\title{
The field of fertility preservation comes of age
}

\author{
David F. Albertini ${ }^{1}$
}

Published online: 19 August 2015

(C) Springer Science+Business Media New York 2015

We welcome our readership to this unique issue of JARG featuring the fast-paced world of fertility preservation. Our treatment of this topic comes from a mix of motivations, not the least of which is to provide our readership with upto-date coverage of the various facets of fertility preservation as advances in this domain continue to influence patterns of patient care. A further stimulus derives from a meeting held earlier at which up and coming young scientists and physicians were invited to present their findings alongside many of the leaders in the field. Most of the papers comprising this special issue of JARG are original contributions from the meeting participants and represent a full spectrum of the topics currently attracting so much attention from patients, clinicians, and scientists alike.

What has piqued the interests of so many in the past decade is the maturation of a range of ARTs intended for protection and/or restoration of fertility threatened in individuals who have undergone gonadotoxic therapies, typically due to cancer. The road to today's array of options has not been a

Capsule Launched as an effort to deal with the threat of sterility in young cancer patients, the field of fertility preservation has opened doors to the diagnosis and treatment of infertility long before it occurs, and for many whose hopes languished with contemporary lifestyles and the compromises that attend them. While technological strides continue to be made, will ethical and societal implications be addressed in time as what was initially perceived as an option for cancer patients is fast becoming a fashion statement for waiting even longer to bear children?

David F. Albertini

DALBERTINI@kumc.edu

1 University of Kansas Medical Center, Kansas, KS, USA straightforward one, nor has it been without controversy given the societal issues attendant to life and death decisions for both male and female patients across the age spectrum. And in retrospect, the pioneering efforts made by Teresa Woodruff and her colleagues in the Oncofertility program at Northwestern University not only brought bench technology to the stage but recognized early on the importance of a communication highway between patients, oncologists, and fertility specialists if ever the needs of this expanding population were to be met.

The range of technologies that have received inspiration in some cases, and scrutiny in others, is well represented by the contributors in the following pages. Whatever technology has emerged de novo or witnessed refinement as a result of the momentum gained through the practical realization of fertility preservation, we most certainly remain in an early stage of maturation that will eventually touch upon ARTs broadly and our fundamental understanding of human reproductive biology.

Towards this end, the reader will find not simply updates on subjects as varied as the ethics of oocyte cryopreservation, or fertility recovery prospects in different disease conditions, but an engaging series of papers on the impacts of ovarian tissue cryopreservation in animal models that reveals properties of the mammalian ovary previously not appreciated. Insights from these studies are being developed to improve the success rates after autologous transplantation in humans. To wit, reported here is the most recent inventory of live births resulting from autologous grafts from cryopreserved ovarian tissues, a procedure that remains deeply embedded in the realm of the experimental.

We welcome your feedback on our humble attempt to bring the JARG readership a step or two closer to the field of fertility preservation and its nascent triumphs and future challenges. 